\title{
LETTER \\ Millimeter-Wave Radar Target Recognition Algorithm Based on Collaborative Auto-Encoder
}

\author{
Yilu MA ${ }^{\dagger \mathrm{a})}$, Zhihui $\mathrm{YE}^{\dagger \mathrm{b})}$, Nonmembers, and Yuehua $\mathrm{LI}^{\dagger \mathrm{c})}$, Member
}

\begin{abstract}
SUMMARY Conventional target recognition methods usually suffer from information-loss and target-aspect sensitivity when applied to radar high resolution range profile (HRRP) recognition. Thus, Effective establishment of robust and discriminatory feature representation has a significant performance improvement of practical radar applications. In this work, we present a novel feature extraction method, based on modified collaborative auto-encoder, for millimeter-wave radar HRRP recognition. The latent frame-specific weight vector is trained for samples in a frame, which contributes to retaining local information for different targets. Experimental results demonstrate that the proposed algorithm obtains higher target recognition accuracy than conventional target recognition algorithms.

key words: target recognition, high resolution range profile, auto-encoder, frame-specific weight vector
\end{abstract}

\section{Introduction}

Target recognition still remains challenging in many practical radar applications. An effective target recognition framework could greatly enhance the recognition ability of radar system. As the high resolution range profile (HRRP) consists of abundant spatial structure information and could be obtained and processed efficiently in millimeter-wave bands, it has been successfully applied to millimeter-wave radar target recognition [1]. The conventional feature extraction algorithms, such as primary component analysis (PCA) and linear discriminant analysis (LDA), usually focus on extracting low-dimensional features from highdimensional domains, which could help to preserve the potential structure of targets. However, the performance of these conventional algorithms degrade as inevitably causing information-loss [2]. Therefore, facing unpredictable information corruption caused by noise interference, and information variation caused by different pitching angle and azimuth, it's crucial to utilize a more effective feature extraction model to build robust and discriminatory representations.

Auto-encoder has been widely applied in many fields and has made superior achievements in recent years. For instance, denoising auto-encoder trains a one-hidden-layer neural network to build robust latent representations from

Manuscript received June 28, 2018.

Manuscript revised September 4, 2018.

Manuscript publicized October 3, 2018.

${ }^{\dagger}$ The authors are with School of Electronic and Optical Engineering, Nanjing University of Science and Technology, Nanjing, China.

a) E-mail: mayilu1991@ njust.edu.cn

b) E-mail: yezh@njust.edu.cn (Corresponding author)

c) E-mail: nlglyh2013@ sina.cn

DOI: $10.1587 /$ transinf.2018EDL8142 partly corrupted input data, and tries to reconstruct the initial clean version [3]. Collaborative filtering $(\mathrm{CF})$ is commonly used in recommender systems [4], and Wu combines denoising auto-encoder with CF theory to improve the performance of the recommender system [5]. Recurrent autoencoder is utilized to perform sequence generation task, and outperforms the traditional methods on both recommendation and sequence generator task [6].

In this work, a novel collaborative target recognition method, based on modified collaborative auto-encoder (CAE), is proposed for millimeter-wave target HRRP recognition. The CAE is exploited for building discriminatory feature representations, and the MLP is utilized for recognition. The main contributions of the recognition framework are summarized as follows: 1) the preference information of the HRRP sample in different azimuth is taken into account when training the CAE, which could help to retain local discriminatory features for HRRP recognition; 2) rather than training a specific weight vector for each HRRP sample, according to the property of HRRP, the samples within a small angular sector share the same weight vector, which is defined as "frame-specific weight vector". As a result, the computation complexity could be reduced when maintaining the advantage of the CAE. Experimental results demonstrate that the proposed algorithm has the capability of building more discriminatory representations for input HRRP samples, and provide a higher recognition accuracy for millimeter-wave radar target recognition than conventional algorithms.

\section{Recognition Algorithm with Collaborative Auto- Encoder}

\subsection{Collaborative Auto-Encoder}

The structure of CAE can be formally illustrated in Fig. 1 . Similar to the standard auto-encoder, the CAE is also represented as a one-hidden-layer neural network. The key difference is that a latent vector related to input sample is trained at the encoding process, which makes this neural network be a much better feature extractor for some specific application.

In the input layer, there are $(I+1)$ units. The first $I$ units are input units, and the last one is defined as samplespecific unit, whose associated weight vector is unique for each sample. In the hidden layer, there are $H$ units which are fully connected with the units in the input layer. Thus, we define $W^{H \times I}$ to denote the associated weight matrix between 


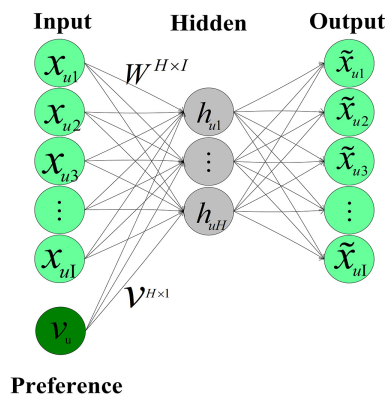

Fig. 1 The structure of CAE.

the input layer and the hidden layer, and $V^{H \times 1}$ to denote the sample-specific weight between sample-specific unit and hidden layer. There are $I$ units in the output layer, which represent the reconstruction of the input sample. We define $b^{H \times 1}$ to represent the bias of encoding operation.

Let $X=\left\{x_{1}, \ldots, x_{N}\right\}$ represent the input dataset, where $x_{i} \in R^{I}$ is an independent sample. CAE first maps the input $x_{i}$ to a latent representation $h_{i}$, which can be computed as follows

$$
h_{i}=f\left(w x_{i}+v_{i}+b\right)
$$

where $f(\cdot)$ represents the activation function of the mapping process, $w$ represents the associated weight matrix, and $b$ is the bias vector, $v_{i}$ represents the sample-specific weight of $x_{i}$.

The decoding operation can be seen as the reconstruction of input data, which is represented as

$$
\tilde{x}_{i}=f\left(w^{\prime} h_{i}+b^{\prime}\right)
$$

where $w^{\prime}$ and $b^{\prime}$ represent the weight matrix and bias vector of the output layer.

Usually, the parameters are learnt via minimizing the following object function.

$$
L=\arg \min \left\{\sum_{i=1}^{N}\left\|\tilde{x}_{i}-x_{i}\right\|^{2}+\|W\|_{2}^{2}+\|V\|_{2}^{2}+\|b\|_{2}^{2}\right\}
$$

The gradient descent is usually applied to optimize the parameters of neural networks. The gradients for parameters of the CAE are represented as follows

$$
\begin{aligned}
& w_{(k+1)}^{\prime}=w_{(k)}^{\prime}+\eta \frac{\partial L}{\partial \tilde{x}} \frac{\partial \tilde{x}}{\partial w^{\prime}} \\
& b_{(k+1)}^{\prime}=b_{(k)}^{\prime}+\eta \frac{\partial L}{\partial \tilde{x}} \frac{\partial \tilde{x}}{\partial b^{\prime}} \\
& w_{(k+1)}=w_{(k)}+\eta \frac{\partial L}{\partial h} \frac{\partial h}{\partial w} \\
& v_{(k+1)}=v_{(k)}+\eta \frac{\partial L}{\partial h} \frac{\partial h}{\partial v} \\
& b_{(k+1)}=b_{(k)}+\eta \frac{\partial L}{\partial h} \frac{\partial h}{\partial b}
\end{aligned}
$$

where $k$ and $\eta$ represent the number of iteration and learning rate, respectively.
Table 1 Training of CAE.

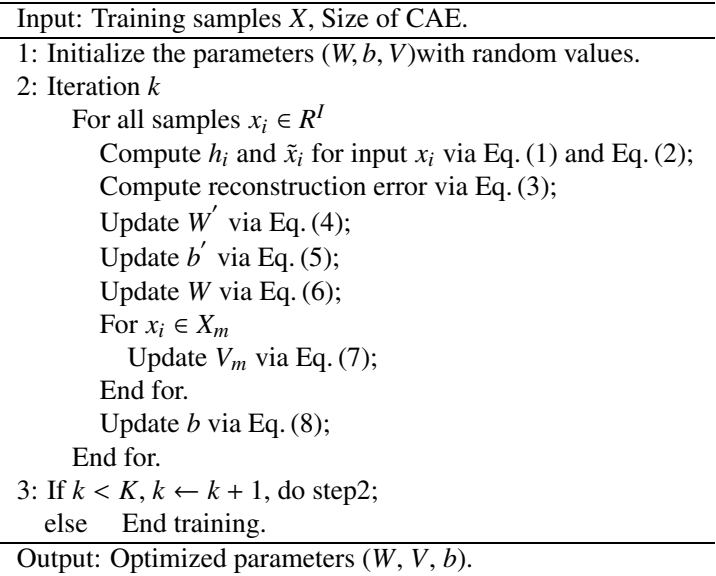

2.2 The Recognition Algorithm for Millimeter-Wave Radar HRRP

In this section, a stable algorithm is designed for millimeterwave target HRRP recognition, which utilizes the pretrained CAE as feature extractor to obtain low-dimensional representations from high-dimensional HRRP data. Compared with conventional feature extractors, the CAE is able to retain the specific details of the different structure. However, we can also observe that with the increase of training samples, the training of sample-specific weight vector will increase the computation complexity. According to the scattering center target model, the HRRP within a small targetaspect sector without scatterers' motion through range cells (MTRC) can be regarded as in a frame, and preserve local invariance. The limitation of the target-aspect change to avoid scatterers' MTRC is represented as

$$
\delta_{\varphi} \leq\left(\delta_{\varphi}\right)_{M T R C} \triangleq \frac{C}{2 B L_{x}}
$$

where $B$ means the bandwidth of the radar signal, $L_{x}$ and $C$ denote the maximum target dimension in cross range and the speed of light, respectively [7].

Thus, when training the CAE, we divide the HRRP samples into $M$ different frames according to different azimuth, and the HRRP in one frame $X_{m}$ share the same weight vector $v_{m}$, which is defined as frame-specific weight vector. In this way, the computation complexity can be decreased, and the advantage of CAE can be preserved.

Suppose that we have $N$ training samples and each sample is an $I \times 1$ vector, the number of hidden units is fixed to $H$. The training samples are divided into $M$ frames and the unsupervised training of CAE is shown in Table 1.

The classifier we used is a multi-layer perception (MLP), which is composed of fully connected units. The classifier is trained with supervised learning method, and the parameters are optimized via back-propagation method.

Noting that for each training sample $x_{i}$, a corresponding frame-specific weight vector $v_{m}$ is trained. Thus, in order 
Table 2 Training of recognition algorithm.

Input: Training samples $X$, Labels $Y$, Size of CAE, Testing samples
1: Initialize the parameters $(W, b, V)$ with random values.
2: Optimize the parameters of CAE according to Table 1.
3: Take the latent representation $h$ as input and label $y$ as output;
Train the MLP classifier via BP method, optimize the parameters.
4: Compute the similarity coefficient via Eq. (10);
select $v_{m}$ via three most relevant $x_{i}$;
encode the latent representation $h_{t e}$ via Eq. (1).
5: Feed $h_{t e}$ to the trained classifier, and calculate the label.
Output: Labels of the testing samples

to select an appropriate weight vector when encoding testing sample $x_{t e}$, we evaluate the similarity between the testing samples and training samples, and based on the max value of similarity, the appropriate weight vector is assigned. The formulation for computing the similarity is represented as

$$
S_{i}=\frac{<x_{t e}, x_{i}>}{\left\|x_{t e}\right\|\left\|x_{i}\right\|}
$$

where $<\cdot>$ represents the inner product operation, and $\|\cdot\|$ represents the modular arithmetic. The index of most relevant training sample is utilized to find the corresponding frame-specific weight vector $v_{m}$ for testing sample $x_{t e}$, and then the representation is fed to the classifier to generate the corresponding label. The procedure of the proposed recognition algorithm is shown in Table 2.

Target-aspect sensitivity is always an important issue in HRRP recognition, which degrades the quality of features extracted by traditional methods. The HRRP samples can be significantly different for single target with variable aspect angles. Therefore, it is tough to obtain discriminatory and uniform feature without solving this issue. Compared with basic AE, rather than training a fixed encoder for all HRRP samples, the proposed CAE model takes the targetaspect sensitivity into consideration to train the complementary frame-specific weight vector. It can be seen as an error correction term to reduce the impact of target-aspect sensitivity, so that to build a more robust feature representation for each target. The inner-class distance could be reduced and the local structure is preserved, thus improving the quality of extracted features.

\section{Experiments and Result Analysis}

High range resolution profile is the amplitude of the coherent sum of the complex time signal returns from the scatters in each range cell. It contains abundant information about the radial location of the scatters and geometric structure, which can be used in target recognition. The proposed algorithm is evaluated on both real HRRP data and simulated HRRP data. To demonstrate its efficiency, the performance of the proposed algorithm is compared with the following 5 approaches: (1) Locality Preserving Projections (LPP); (2) Linear Local Tangent Space Alignment (LLTSA); (3) Neighborhood Preserving Embedding (NPE); (4) kernal PCA (KPCA); (5) Auto-encoder (AE). The kernal function of KPCA is radial basis function (RBF), and 18

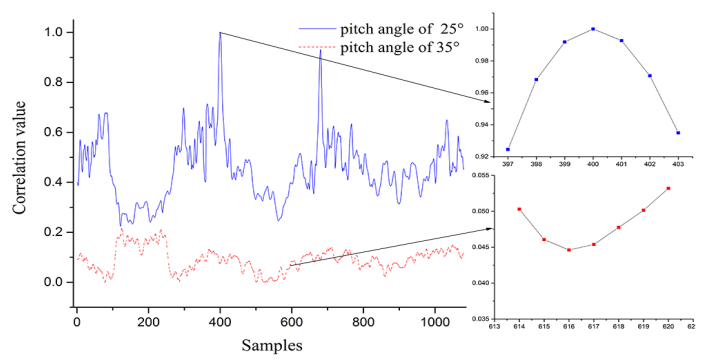

Fig. 2 The correlations between the testing sample and training samples.

features are retained. The dimensions of LPP, LLTSA and NPE are fixed to 20,15 and 15, respectively.

\subsection{Experimental Results on Simulated Data}

The proposed algorithm is first evaluated on the simulated data. The frequency of the simulated radar system is fixed to $35 \mathrm{GHz}$, and the bandwidth is fixed to $500 \mathrm{MHz}$. The simulated scatters are placed in different positions to simulate 3 different targets. The azimuth varies from $0^{\circ}$ to $180^{\circ}$ with the step of $0.5^{\circ}$, and the pitch angle varies from $25^{\circ}$ to $35^{\circ}$ with the step of $5^{\circ}$. Finally, 3240 HRRP samples are generated. For single simulated target in one pitch angle, its HRRPs within $2^{\circ}$ can be regarded as one frame and share the preference weight vector. 1800 samples are selected as training samples, and the rest samples are utilized to evaluate the performance. The input units are fixed 256 and 257 for $\mathrm{AE}$ and $\mathrm{CAE}$, and 20 for the output. The learning rate is set as 0.8 , and the batch size is 10 with 50 training epochs.

Figure 2 shows the normalized correlations between the selected HRRP sample (the 400th HRRP from pitch angle of $25^{\circ}$ ) and the others in the dataset. The first 360 values represent the correlations of target 1, the following 360 values represent the correlations of target 2 , and the 360 last values represent the correlations of target 3 . It can be observed that the correlation changes with the variation of azimuth, which reflects target-aspect sensitivity. The samples from different pitch angles keep discriminatory. On the other hand, as shown in the top right of Fig. 2, the correlation maintains stable within a small angular range. Thus, it is reasonable to train the frame-specific weight vector for a frame within certain azimuth, which helps to retain the discriminatory information. Compared with training a preference weight for each sample, it also decreases computational complexity and reduces the risk of overfitting. The most suitable weight vector for the testing sample is assigned according to the similarity (correlations value).

The SVM and the MLP classifier are applied to perform the recognition experiments, and the results are shown in Table 3. Among the four conventional algorithms, LPP, LLSTA and NPE obtain higher accuracies than KPCA for both SVM and MLP classifier, which indicates that manifold learning methods are effective for learning better feature representations. On the other hand, it is surprising to see that the MLP achieves higher accuracy than the SVM. 
Table 3 The accuracies of the simulated data.

\begin{tabular}{lcc}
\hline Methods & SVM & MLP \\
\hline LPP & 91.17 & 90.89 \\
LLSTA & 83.77 & 84.53 \\
NPE & 90.41 & 91.25 \\
KPCA & 79.37 & 81.38 \\
AE & 94.06 & 95.97 \\
CAE & 96.39 & 98.54 \\
\hline
\end{tabular}

The only feature extraction algorithm based on which SVM achieves better result is LPP, but the performance gains is not significant. In general, the algorithms based on autoencoder perform better than conventional ones, especially when feeding the extracted features to the MLP. One reasonable explanation is that, we connect the CAE (AE) with the MLP when training the MLP classifier via supervised learning method, and the parameters of CAE (AE) are further optimized for MMW radar HRRP recognition. Thus, the recognition accuracies of the AE-MLP and the CAEMLP model are higher than the AE-SVM and the CAESVM model. The main difference between CAE and AE is the frame-specific weight vector, which is designed to be a compensation term for extracted feature. Compared with the result of $\mathrm{AE}$, the frame-specific weight vector shows its advantage of retaining the discriminatory structure information, thus improving the recognition accuracy.

\subsection{Experimental Results on Real Data}

The CAE based algorithm is then evaluated on the measured data, and the experimental environment is as follows. Based on the theory that a complex target can be regarded as the combination of scattering points, we use a number of corner reflectors settled at different positions to simulate three different targets. The distance from the radar to the center of simulated target is 10 meters. The background is cement floor. The radar system works at $24 \mathrm{GHz}$ and the bandwidth of transmit signal is $400 \mathrm{MHz}$. We gradually change the azimuth from $0^{\circ}$ to $180^{\circ}$ to measure each target, with the step of $1^{\circ}$. Thus, each target has 180 HRRPs, and each HRRP is a $256 \times 1$ vector after processing. The input units for $\mathrm{AE}$ and CAE are also 256 and 257, and 20 for the output, while the batch size is 5 with 50 training epochs and the learning rate is 0.5 .

The recognition rsults of all algorithms are shown in Table 4. In gerneral, the results of the MLP and the SVM are consistent. The manifold learning methods (LPP and NPE) have the similar performance, and performs better than KPCA. Compared with other four methods, the AE and CAE also achieve higher accuracy, but it also takes a longer time for the $\mathrm{AE}$ and $\mathrm{CAE}$ to train the parameters. Besides, we can find that the CAE based algorithm achieves the highest recognition accuracy in the experiment of real data, which indicates that the proposed algorithm has the capability of build better feature representations for MMW HRRPs than conventional algorithms.

Compared with the results of simulated data, we can observe that the accuracy of real data is little lower. It may
Table 4 The accuracies of the measured data.

\begin{tabular}{lll}
\hline Methods & SVM & MLP \\
\hline LPP & 87.31 & 89.25 \\
LLSTA & 82.65 & 84.35 \\
NPE & 85.12 & 87.16 \\
KPCA & 77.68 & 78.49 \\
AE & 93.42 & 94.55 \\
CAE & 94.92 & 95.65 \\
\hline
\end{tabular}

be explained that although we have taken noise into account for HRRP simulation, the real data is obtained from more complex scenarios, and the number of training samples is also less than simulated ones.

\section{Conclusions}

In this paper, a collaborative auto-encoder based feature extraction algorithm is proposed to build the more discriminatory and robust representation for millimeter-wave radar HRRP. Frame-specific weight vectors are trained to benefit the encoding of the HRRP samples. Experimental results on both simulated data and real data demonstrate that the CAE based algorithm can provide higher recognition accuracy than conventional algorithms. Further research will focus on decreasing the computational complexity of training frame-specific weight vectors.

\section{Acknowledgments}

This work is supported by National Natural Science Foundation of China (61601237), Jiangsu Provincial Natural Science Foundation of China (BK20160901), Natural Science Foundation of Jiangsu Higher Education Institutions of China (16KJB420001).

\section{References}

[1] J. Wang, Y. Li, and J. Chen, "Local reconstruction error alignment: A fast unsupervised feature selection algorithm for radar target clustering," IEICE Trans. Inf. \& Syst., vol.E97-D, no.2, pp.357-360, Feb. 2014.

[2] B. Chen, H. Liu, and Z. Bao, "PCA and kernel PCA for radar high range resolution profiles recognition," IEEE International Radar Conference, pp.528-533, 2005.

[3] P. Vincent, H. Larochelle, Y. Bengio, and P.-A. Manzagol, "Extracting and composing robust features with denoising autoencoders," International Conference on Machine Learning, pp.1096-1103, ACN, 2008.

[4] Q. Li, X. Zheng, and X. Wu, "Collaborative autoencoder for recommender systems," 2017.

[5] Y. Wu, C. DuBois, A.X. Zheng, and M. Ester, "Collaborative denoising auto-encoders for Top-N recommender systems," ACM International Conference on Web Search and Data Mining, pp.153-162, 2016.

[6] H. Wang, X. Shi, and D.Y. Yeung, "Collaborative recurrent autoencoder: Recommend while learning to fill in the blanks," 2016.

[7] B. Feng, B. Chen, P.H. Wang, et al., "Feature extraction method for radar high resolution range profile targets based on robust deep networks," Dianzi Yu Xinxi Xuebao/Journal of Electronics Information Technology, vol.36, no.12, pp.2949-2955, 2014.

[8] P. Mian, J. Jie, L. Zhu, C. Jing, and Z. Tao, "Radar HRRP recognition based on discriminant deep autoencoders with small training data size," Electron. Lett.. vol.52, no.20, pp.1725-1727, 2016. 